\title{
Relativity in fundamental astronomy
}

\author{
M. Soffel $\dagger$ \\ Lohrmann Observatory, Dresden Technical University, 01062 Dresden, Germany
}

\begin{abstract}
An overview is given over the broad field of Relativity in Fundamental Astronomy. The present status is recalled and deficiencies are pointed out that might lead to future work within IAU Commission 52.
\end{abstract}

Keywords. relativity, gravitation, reference systems, astrometry, ephemerides

The field of Relativity in Fundamental Astronomy comprises, at least, relativity in i) astronomical space-time reference systems, ii) in celestial mechanics, iii) in astrometry, and iv) in metrology. In addition, it concerns certain astronomical concepts; the ecliptic is an example.

In the field of astronomical reference systems, the Barycentric and Geocentric Celestial Reference Systems, BCRS and GCRS, were adopted by IAU 2000 resolutions (e.g., Soffel et al., 2003). They were constructed in the 1st post-Newtonian approximation to Einstein's theory of gravity and all matter and cosmic energy outside the solar system was neglected, as was the influence of cosmic expansion. In Klioner \& Soffel (2004) it was shown that the cosmic expansion has practically no influence on local physics, e.g. solar system ephemerides. Associated time scales have been discussed in great detail. First attempts to construct similar reference systems at the 2nd post-Newtonian approximation for astrometry have been published (e.g., Minazzoli \& Chauvineau, 2009). Topocentric Celestial Reference systems, either directly related with the BCRS or coming from the GCRS with direct relation to the ITRS, have been constructed.

Every modern solar system ephemeris uses the post-Newtonian (PN) dynamical equations for mass monopoles (EIH equations). In the literature one finds discussions of spin-orbit and spin-spin couplings, the translational equations of motion with all mass and spin multipole moments as well as rotational equations of motion to post-Newtonian (PN) order (e.g., Damour et al., 1991-1994). For practical applications such as a postNewtonian treatment of Earth's rotation a PN model of rigidly rotating multipoles has been worked out as a starting point for further perturbative treatments (Klioner et al., 2001). The dynamics of two mass monopoles (point masses) has been worked out to order $1 / c^{7}$ (3.5 PN) (e.g., Königsdörffer et al., 2003). For satellite motion relativistic potential coefficients have been adopted by IAU2000 resolutions. Relativistic inertial forces (LenseThirring and geodesic precession/nutation) have been discussed extensively, relativistic tidal forces have been discussed in Damour et al. (1993).

In the field of astrometry the relativistic model of Klioner (2003) has been refined over the years. It is basically a coordinate picture but it has been demonstrated in Klioner (2004) that with a choice of adequate local coordinates moving with the observer, results agree with those of a tetrad formalism, i.e., they are coordinate-independent. The intrinsic accuracy of the Klioner model is of order $0.1 \mu$ as.

In the field of relativity in metrology we face the following issues: clock synchronization and time dissemination, GPS - GLONASS - GALILEO, VLBI, SLR - LLR, Laser Gyros, Doppler measurements and Pulsar Timing. The problem of clock synchronization and

$\dagger$ E-mail: michael.soffel@tu-dresden.de 
time dissemination by means of electromagnetic signals has been discussed extensively in the literature (e.g., Klioner 1991; Wolf \& Petit, 1995; Petit \& Wolf, 2005). The intrinsic accuracy here is below 1 ps. For the problem of relativity in GPS (GLONASS GALILEO) the standard reference is Ashby (2003). The present relativistic VLBI model is a consensus model (Eubanks 1991) with an accuracy of about 1 ps. A post-Newtonian treatment of SLR and LLR can be found in many places (e.g., Soffel 1989). A ring-laser gyroscope consists basically of a closed tube in which laser activity is excited so that two laser beams, one traveling in clockwise the other one in counter-clockwise direction, interfere behind a beam splitter where the interference fringes can be analyzed. Such a gyroscope is an inertial device. In contrast to the geodetic space techniques it is sensitive to the instantaneous rotation vector of the Earth. A post-Newtonian theory of ring-laser gyroscopes can be found in Scully et al. (1981), Soffel (1989) and Bosi et al. (2011). Relativity terms are of order $7 \times 10^{-10}$, far below the present level of achievable accuracies. Martin et al. (1985) is the classical paper where relativity in Doppler measurements to spacecraft is treated. A large number of papers is devoted to a relativistic description of pulsar timing (e.g., Blandford et al. 1976; Haugan 1985, Damour \& Taylor 1992; Kopeikin 1999).

Comparing the intrinsic theoretical accuracies with presently achievable ones in observations/measurements one finds that present relativistic models seem to be sufficient for the forthcoming decades. Nevertheless, there is the wish to work out a relativistic VLBI model with an accuracy of about 0.1 ps (presently the accuracy of VLBI observations is of order of a few ps); it should be consistent and 'complete' at this level and well documented. The concept of a post-Newtonian rigidly rotating multipole model as a useful starting point for a relativistic description of global geodynamics should be critically examined.

\section{References}

Ashby, N. 2003, Living Rev. Relativity 6, 1

Blandford, R. \& Teukolsky, S. 1978, Astrophys. J. 295, 580

Bosi, F., et al. 2011, Phys.Rev. D84, 122002

Damour, T., Soffel, M., Xu, C. 1991-1994, Phys.Rev. D43, 3273 (1991); D45, 1017 (1992); D47, 3124 (1993); D49, 618 (1994)

Damour, T. \& Taylor, J. 1992, Phys.Rev. D45, 1840

Eubanks, T. M. 1991, Proc. of the U.S. Naval Observatory Workshop on Relativistic Models for Use in Space Geodesy, USNO

Haugan, M. 1985, Astrophys. J. 296, 1

Klioner, S. 1991, Celest.Mech. 53, 81

Klioner, S. 2003, Astron. J. 125(3), 1580

Klioner, S. 2004, Phys. Rev. D69, 124001

Klioner, S., Soffel, M., Xu, C., \& Wu, X. 2001, In: Proceedings of Journées'2001, N. Capitaine (ed.), Paris Observatory, Paris, 232; arXiv:astro-ph/0303376

Klioner, S. \& Soffel, M. 2004, ESA SP-576, 305; arXiv:astro-ph/0411363v2

Königsdörffer, C., Faye, G., \& Schäfer, G. 2003, arXiv:gr-qc/0305048v2

Kopeikin, S. 1999, Mon. Not. R. Astron. Soc. 305, 563

Martin, C., Torrence, M., \& Misner, C. 1985, J.Geophys.Res. 90, 9403

Minazzoli, O. \& Chauvineau, B. 2009, Phys.Rev. D79, 084027

Petit, G. \& Wolf, P. 2005, Metrologia 42, S138

Scully, M., Zubairy, M., \& Haugan, M. 1981, Phys.Rev. A24, 2009

Soffel, M. 1989, Relativity in Astrometry, Celestial Mechanics and Geodesy, Spinger, Berlin

Soffel et al. 2003, Astron. J., 126, 2687

Wolf, P. \& Petit, G. 1995, Astron. Astrophys. 304, 653 